\title{
Prasugrel for the treatment of patients with acute coronary syndrome
}

\section{Filippo Marzot \\ Vittorio Pengo}

Clinical Cardiology, Thrombosis

Centre, University of Padua, Italy
Correspondence: Vittorio Pengo Clinical Cardiology, Thrombosis Centre, Via Giustiniani 2, 35I 28 Padova, Italy $\mathrm{Tel} / \mathrm{Fax}+39$ (0) 498215658 Email vittorio.pengo@unipd.it

\begin{abstract}
Prasugrel (CS-747, LY640315) is a novel member of the thienopyridine class of oral anti-platelet agents (also including ticlopidine and clopidogrel). Like other thienopyridines, prasugrel is a prodrug that is inactive in vitro. Prasugrel's conversion to its active metabolite is more rapid and efficient than that of other thienopyridines, with a less strict dependence on specific cytochrome P-450 enzymes. Prasugrel's active metabolite (R-138727) binds specifically and irreversibly to the platelet P2Y12 purinergic receptor, thus inhibiting ADP-mediated platelet activation and aggregation. Preclinical data and early clinical data in healthy subjects showed greater platelet inhibition and consistency with prasugrel as opposed to clopidogrel. Clinical studies in patients with cardiovascular disease confirmed the greater efficacy of prasugrel compared with clopidogrel. Collectively, these phase $1 / 1 \mathrm{~b}$ studies and a phase 2 study (JUMBO-TIMI 26) aided in dose selection for the phase 3 trial (TRITONTIMI 38) in patients with acute coronary syndrome undergoing percutaneous coronary intervention. This trial once again confirmed the greater anti-platelet effect of prasugrel, but also highlighted a higher risk of bleeding, even fatal. Another phase 2 trial (PRINCIPLE-TIMI 44) compared prasugrel and high-dose clopidogrel in patients undergoing cardiac catheterization for planned PCI.
\end{abstract}

Keywords: prasugrel, oral antiplatelet agents, acute coronary syndrome

\section{Platelets and atherothrombosis}

Platelets are one of the main actors in the spontaneous process of atherothrombosis and in post-coronary stenting acute thrombosis. ${ }^{1}$ Following the initial adhesion of platelets to collagen, von Willebrand factor and other subendothelial proteins in the site of vascular injury or ruptured atherosclerotic plaque, platelet activation maintains itself through the release of adenosine diphosphate (ADP), thromboxane $\mathrm{A}_{2}\left(\mathrm{TXA}_{2}\right)$ and other mediators. The interaction of ADP with the G protein-coupled purinergic receptors, $\mathrm{P} 2 \mathrm{Y}_{1}$ and $\mathrm{P} 2 \mathrm{Y}_{12}$, leads to further activation of platelet functions, such as aggregation, shape change and secretion. Activation of this process in coronary arteries leads to the formation of platelet-rich thrombi, which in turn initiates the cascade of vascular occlusion, tissue ischemia and myocardial necrosis that is known under the collective name of acute coronary syndrome (ACS).

Also, when patients with coronary atherosclerotic disease are treated with percutaneous coronary intervention (PCI), there is a significant risk of platelet activation and aggregation at the site of balloon dilation and stenting, which could lead to stent-associated thrombosis (SAT). ${ }^{2}$ Accordingly, antiplatelet drugs have been shown to be useful in reducing such complications. ${ }^{3,4}$

According to this, anti-platelet agents are widely used for prevention and therapy in patients with atherothrombotic coronary disease, and to prevent SAT after performing PCI. 


\section{Currently used thienopyridines}

Currently used thienopyridines, ticlopidine and clopidogrel, are both prodrugs that need to be converted to an active metabolite that binds irreversibly to the $\mathrm{P} 2 \mathrm{Y}_{12}$ receptor, thus preventing ADP binding and ADP mediated platelet activation and aggregation. ${ }^{5}$ Of the two, clopidogrel is the more commonly used, due to its improved tolerability and lower incidence of hematologic side-effects. Numerous studies have demonstrated the safety and efficacy of dual antiplatelet therapy - aspirin co-administered with either ticlopidine or clopidogrel - in improving the clinical outcome of patients with ACS and reducing the risk of early SAT in patients undergoing PCI (clopidogrel being a better choice for the latter, by virtue of its greater safety and tolerability over ticlopidine). Large trials such as CREDO or PCI-CURE confirmed the superiority of dual antiplatelet therapy (aspirin and clopidogrel) in preventing major cardiovascular events over aspirin and placebo. ${ }^{3,4}$ However, clopidogrel exhibits some limitations such as modest antiplatelet activity, a significant interpatient variability and delayed onset of action. Some clinical evidence suggests that patient with reduced response to clopidogrel (even at higher loading dose regimens) are at increased risk of major cardiovascular events and SAT after undergoing PCI. ${ }^{6,7}$

\section{Investigational agents}

Prasugrel (CS-747, LY640315) is a novel investigational drug belonging to the thienopyridines class of antiplatelet agents. ${ }^{8}$ In preclinical and clinical studies it has demonstrated to be an orally active inhibitor of platelet activation and aggregation, having more potent activity, lesser interpatient variability and faster onset of action as compared to clopidogrel and ticlopidine. Initial testing in rats demonstrated that prasugrel produced dose-related inhibition of platelet activity with a potency approximately 10- and 100-fold greater than clopidogrel and ticlopidine, respectively. This can be related to more efficient formation of prasugrel's active metabolite as opposed to that of clopidogrel. The active metabolites of both drugs not only inhibit ADP-mediated platelet aggregation, but also other consequences of platelet activation, such as GP IIb/IIIa activation, platelet-leukocyte aggregate formation, platelet procoagulant activity, and inhibition of platelet thromboinflammatory markers. ${ }^{9}$

\section{Pharmacology}

Prasugrel first undergoes rapid hydrolysis by esterases, thus forming thiolactone (R-95913), which is in turn oxidized by intestinal and hepatic cythochrome P 450 (CYP), which causes the opening of the ring structure and the formation of the active metabolite (R-138727), which contains an $-\mathrm{SH}$ group. The active metabolite becomes detectable in plasma after 15 minutes, and reaches peak concentration at 30 minutes after dosing. The main pathway for the elimination of the active metabolite in humans is renal excretion. ${ }^{10}$ The main cytochrome isoenzymes responsible for the formation of the active metabolite are CYP3A and CYP2B6, with lesser contribution by CYP2C9 and CYP2C19. ${ }^{11}$ The latter two, on the other hand, play a major role in the formation of clopidogrel's active metabolite; this would explain why common loss-of-function mutations in these loci greatly affects clopidogrel while barely interferring with the formation of prasugrel's active metabolite. ${ }^{12}$ Furthermore, co-administration of potent CYP3A inhibitors such as ketoconazole, albeit significantly reducing R-138727 maximum concentration, does not affect the area under the time-concentration curve nor prasugrel's antiplatelet effects. ${ }^{13}$ Prasugrel's metabolites do not appear to inhibit cythochromemediated metabolism of other co-administered drugs.

\section{Phase I clinical trials}

Three early phase 1 trials confirmed the results of the preclinical studies on animals, finding prasugrel to be 10 times more potent than clopidogrel, and consistently so, as opposed to clopidogrel's significant variability in response. ${ }^{14-16}$ These results were further proved by a crossover study in healthy, aspirin-free subjects, which found prasugrel to reach higher levels in the active metabolite concentration, thus justifying the greater inhibition of platelet aggregation. Another interesting finding was that clopidogrel non-responders had good response to prasugrel when crossed over. ${ }^{17}$ Another trial, ${ }^{18}$ performed on healthy subjects, had the trial drug co-administered with aspirin: once again, prasugrel proved to be more potent and produced greater inhibition of platelet aggregation. Overall, at the dosing regimens tested, prasugrel was well tolerated, hematomas and minor bleeding being the most reported adverse events. No evidence of hepatic or central nervous system toxicity, neutropenia, thrombocytopenia or QT-prolongation were reported.

The first patient study was a phase $1 \mathrm{~b}$ dose-ranging study of prasugrel versus clopidogrel in patients with stable coronary artery disease already taking aspirin. ${ }^{19}$ Prasugrel loading doses of 40 and $60 \mathrm{mg}$ produced significantly higher inhibition of platelet aggregation than clopidogrel loading dose $(300 \mathrm{mg}$ ), and maintenance dosing maintained stable, dose-dependent inhibition of platelet aggregation that were 
significantly higher than clopidogrel maintenance dose of $75 \mathrm{mg}$ for the prasugrel 10 and $15 \mathrm{mg}$ maintenance dosing. Safety was comparable in the two groups: although an increase in minor bruising and bleeding events was found in the prasugrel $15 \mathrm{mg}$ maintenance dosing group, a posthoc analysis found no relation between higher inhibition of platelet aggregation and bleeding; the study, however, was not powered to detect such differences.

\section{Phase 2 clinical trials}

JUMBO-TIMI 26 was a phase 2 randomized, dose-ranging, double-blind safety trial. 904 patients undergoing elective or urgent PCI were randomized to either receive standard clopidigrel loading and maintenance doses or one of three different prasugrel dosing regimens (40 mg loading dose $/ 7.5 \mathrm{mg}$ maintenance dose, $60 \mathrm{mg} \mathrm{LD} / 10 \mathrm{mg}$ MD or $60 \mathrm{mg} \mathrm{LD} / 15 \mathrm{mg}$ MD) in co-administration with aspirin. ${ }^{20}$ Patients were then monitored for a 30-days follow-up period for bleeding and major adverse cardiac events. Treatment with prasugrel resulted in an overall rate of bleeding comparable to that of patients treated with clopidogrel, although an increased rate of TIMI minimal bleeding events was reported for the high dose prasugrel group as opposed to all the other treatment groups. Treatment with prasugrel attained a non-significantly lower rate of cardiac adverse events (secondary efficacy endpoint), primarily driven by a reduction in myocardial infarction and urgent reintervention. This study served as a foundation for a large phase 3 trial, designed to assess both efficacy and safety.

\section{Phase 3 clinical trials}

TRITON-TIMI 38 was a phase 3 randomized double-blind trial. 13,608 patients with an ACS (unstable angina, NSTEMI or STEMI) undergoing scheduled PCI were randomized to receive either clopidogrel (standard dosing regimen) or prasugrel (60 mg loading dose and $10 \mathrm{mg}$ od maintenance dose) in addition to aspirin. ${ }^{21}$ The primary efficacy endpoint was a composite of death from cardiovascular causes, nonfatal myocardial infarction and non-fatal stroke during the follow up period, while the primary safety endpoints were TIMI life-threatening, major or minor bleeding not related to coronary artery bypass grafting. Endpoints were evaluated at hospital discharge, 30 days, 90 days and then every 3 months for up to a 15 months follow up period. Treatment with prasugrel resulted in a significantly reduced rate of primary efficacy endpoint events (781 in the clopidogrel group, $12.1 \%$, versus 643 in the prasugrel group, 9.9\%; hazard ratio $0.81 ; 95 \% \mathrm{CI} 0.71$ to $0.96 ; \mathrm{p}<0.001)$, especially due to a reduction in myocardial infarction. This equals a $2.2 \%$ absolute reduction and a $19 \%$ relative reduction of primary efficacy endpoints in the prasugrel treatment group. The rate of primary safety endpoints, however, was significantly higher in patients who received treatment with prasugrel (146 in the prasugrel group, 2.4\%, versus 111 in the clopidogrel group, $1.8 \%$; hazard ratio $1.32 ; 95 \% \mathrm{CI}$ 1.03 to $1.68 ; \mathrm{p}=0.03)$, including fatal $(0.4 \%$ versus $0.1 \%$, $\mathrm{p}=0.002$ ) and life-threatening bleeding. The rates of certain efficacy and safety endpoints were included in a prespecified analysis of net clinical benefit (defined as the rate of death from any cause, non-fatal myocardial infarction, non-fatal stroke, or non-CABG-related non-fatal TIMI major bleeding) and the findings were in favor of prasugrel $(13.9 \%$ of patients in the clopidogrel group vs $12.2 \%$ in the prasugrel group; hazard ratio, $0.87 ; 95 \% \mathrm{CI}, 0.79$ to $0.95 ; \mathrm{p}=0.004$ ). Post-hoc analyses were then performed to identify the subgroups of patients who did not have a net clinical benefit from the use of prasugrel and those who had net clinical harm. Patients with clinical history of stroke or transient ischemic attack had net clinical harm: they had no evidence of clinical benefit as evaluated by primary efficacy endpoints, while exhibiting a significant trend toward major bleeding (including intracranial hemorrhage). Patients 75 years of age or older and patients weighing less than $60 \mathrm{~kg}$ had no net clinical benefit, although they did not have net clinical harm either; the bleeding rate in these two categories of patients was greater, although not statistically significant. Among patients who did not fall in any of these three categories, prasugrel exhibited greater efficacy than clopidogrel, while the bleeding rate was comparable among the two.

\section{Comparison with higher clopidogrel dosing regimens}

PRINCIPLE-TIMI 44 was a phase 2 randomized, double-blind, 2-step crossover study in which prasugrel was compared with high-dose clopidogrel in patients undergoing cardiac catheterization for planned PCI. ${ }^{22}$ Patients were randomized to receive either a clopidogrel $600 \mathrm{mg}$ or prasugrel $60 \mathrm{mg}$ loading dose one hour before undergoing heart catheterization. After PCI, patients were then assigned to receive a once-daily maintenance dose of either clopidogrel $150 \mathrm{mg}$ or prasugrel $10 \mathrm{mg}$, according to the assigned treatment group, for a period of 14 days. From day 15 , after data collection and endpoint evaluation, they were then switched to receive the alternate maintenance therapy for an additional 14 days. Inhibition of platelet aggregation was tested both after the loading dose and during 
the maintenance period. Treatment with prasugrel resulted in a quicker ( $<30 \mathrm{~min})$ and persistently higher inhibition of platelet aggregation, after the loading dose and during all of the treatment period.

\section{Unpublished and ongoing trials}

Further clinical trials have been, or are being conducted to establish an advantage of prasugrel over clopidogrel by means of its pharmacodynamic effects (inhibition of platelet aggregation measured by various laboratory assays), to evaluate safety and tolerability of switching treatment from clopidogrel to prasugrel and to further assess dosing regimens of both drugs.

\section{Conclusions}

Prasugrel is a potent novel thienopyridine antiplatelet agent that preclinical trials so far have shown to achieve greater, faster and less variable response in terms of inhibition of platelet aggregation as compared with clopidogrel due to more efficient generation of the active metabolite. Clinical trials, in turn, have shown prasugrel to be superior to clopidogrel in preventing major cardiac adverse events in patients with ACS undergoing PCI, while attaining a lesser rate of non-responders. On the other hand, the TRITON-TIMI 38 trial has shown that treatment with prasugrel bears a significant increase in the bleeding rate, including major, life-threatening and fatal events. This raises an obvious safety issue, and although post-hoc analyses seem to have identified those categories of patients that are at increased risk for bleeding, this point needs to be further investigated.

\section{Disclosures}

The authors have no conflicts of interest to disclose.

\section{References}

1. Ruggeri ZM. Platelets in atherothrombosis. Nat Med. 2002;8: 1227-1234.

2. Gurbel PA, Bliden KP, Guyer K, et al. Platelet reactivity in patients and recurring events post-stenting. Results of the PREPARE POSTSTENTING study. J Am Coll Cardiol. 2005;46:1820-1826.

3. Steinhubl PB, Berger JT, Mann III, et al. Early and sustained dual oral antiplatelet therapy following percutaneous coronary intervention: A randomized controlled trial. JAMA. 2002;288:2411-2420.

4. Mehta SR, Yusuf S, Peters RJ, et al. Effects of pretreatment with clopidogrel and aspirin followed by long-term therapy in patients undergoing percutaneous coronary intervention: The PCI-CURE study. Lancet. 2001;358:527-533.

5. Billet NH. Antiplatelet agents and arterial thrombosis. Cardiol Clin. 2008;26:189-201.

6. Hochholzer W, Trenk D, Bestehorn HP, et al. Impact of the degree of peri-interventional platelet inhibition after loading with clopidogrel on early clinical outcome of elective coronary stent placement. $J$ Am Coll Cardiol. 2006;48:1742-1750.
7. Buonamici P, Marcucci R, Migliorini A, et al. Impact of platelet reactivity after clopidogrel administration on drug-eluting stent thrombosis. J Am Coll Cardiol. 2007;49:2312-2317.

8. Niitsu Y, Jakubowski JA, Sugidachi A, Asai F. Pharmacology of CS-747 (prasugrel, LY640315), a novel, potent antiplatelet agent with in vivo $\mathrm{P} 2 \mathrm{Y}_{12}$ receptor antagonist activity. Semin Thromb Hemost. 2005;31:184-194.

9. Sugidachi A, Asai F, Ogawa T, Inoue T, Koike H. The in vivo pharmacological profile of CS-747, a novel antiplatelet agent with platelet ADP receptor antagonist properties. Br J Pharmacol. 2000;129:1439-1446.

10. Farid NA, Smith RL, Gillespie TA, et al. The disposition of prasugrel, a novel thienopyridine, in humans. Drug Metab Dispos. 2007;35:1096-1104.

11. Rehmel JL, Eckstein JA, Farid NA, et al. Interactions of two major metabolites of prasugrel, a thienopyridine antiplatelet agent with the cytochromes P450. Drug Metab Dispos. 2006;34:600-607.

12. Brandt JT, Close SL, Iturria SJ, et al. Common polymorphisms of CYP2C19 and CYP2C9 affect the pharmacokinetic and pharmacodynamic response to clopidogrel but not prasugrel. J Thromb Haemost. 2007;5:2429-2436.

13. Farid NA, Payne CD, Small DS, et al. Cytochrome P450 3A inhibition by ketoconazole affects prsaugrel and clopidogrel pharmacokinetics and pharmacodynamics differently. Clin Pharmacol Ther. 2007; 81:735-741.

14. Asai F, Jakubowski JA, Naganuma H, et al. Platelet inhibitory activity and pharmacokinetics of prasugrel (CS-747) a novel thienopyridine P2Y ${ }_{12}$ inhibitor: A single-ascending dose study in healthy humans. Platelets. 2006;17:209-217.

15. Jakubowski JA, Matsushima N, Asai F, et al. A multiple dose study of prasugrel (CS-747), a novel thienopyridine $\mathrm{P}_{2} \mathrm{Y}_{12}$ inhibitor, compared with clopidogrel in healthy humans. Br J Clin Pharm. 2006;63:421-430.

16. Matsushima N, Jakubowski JA, Asai F, et al. Platelet inhibitory activity and pharmacokinetics of prasugrel (CS-747) a novel thienopyridine P2Y $\mathrm{Y}_{12}$ inhibitor: A multiple-dose study in healthy humans. Platelets. 2006;17:218-226.

17. Brandt JT, Payne CD, Wiviott SD, et al. A comparison of prasugrel and clopidogrel loading doses on platelet function: Magnitude of platelet inhibition is related to active metabolite formation. Am Heart J. 2007; 153:66.e9-e16.

18. Jakubowski JA, Payne CD, Weerakkody GJ, et al. Dose-dependent inhibition of human platelet aggregation by prasugrel and its interaction with aspirin in healthy subjects. J Cardiovasc Pharmocol. 2007;49: 167-173.

19. Jernberg T, Payne CD, Winters KJ, et al. Prasugrel achieves greater inhibition of platelet aggregation and a lower rate of non-responders compared with clopidogrel in aspirin-treated patients with stable coronary artery disease. Eur Heart J. 2006;27:1166-1173.

20. Wiviott SD, Antman EM, Winters KJ, et al. Randomized comparison of prasugrel (CS-747,LY640315), a novel thienopyridine P2 ${ }_{12}$ antagonist, with clopidogrel in percutaneous coronary intervention: Results of the Joint Utilizationì of Medications to Block Platelets Optimally (JUMBO)-TIMI 26 trial. Circulation. 2005;111:3366-3373.

21. Wiviott SD, Braunwald E, McCabe $\mathrm{CH}$, et al. A clinical trial of prasugrel vs. clopidogrel in patients with acute coronary syndromes. $N$ Engl J Med 2007;357:2001-2015.

22. Wiviott SD, Trenk D, Frelinger AL, et al; PRINCIPLE-TIMI 44 Investigators. Prasugrel compared with high loading- and maintenancedose clopidogrel in patients with planned percutaneous coronary intervention: the Prasugrel in Comparison to Clopidogrel for Inhibition of Platelet Activation and Aggregation-Thrombolysis in Myocardial Infarction 44 trial. Circulation. 2007;116:2923-2932. 